\section{Concepts in Toxicology}

by John H. Duffus, Douglas M. Templeton, and Monica Nordberg

Royal Society of Chemistry, Cambridge, 2009. (ISBN 978-0-85404-157-2)

The objective of this book is to give clear explanations of the meaning and usage of key toxicological terms. A need was identified to go beyond glossary definitions (already published in Duffus, Nordberg, and Templeton, 2007) ${ }^{1}$ and make plain the underlying assumptions behind terms that are becoming ever more important in communications relating to chemical safety.

With the advent of antibiotics and increasingly effective means of controlling infectious disease, attention turned to adverse health effects resulting from exposure to toxic substances. Prevention of these adverse effects required regulation of potentially harmful exposures. When this was realized, new laws, such as the Toxic Substances Control Act in the USA, were introduced. These laws required assessment of toxicity and legal definitions of what constituted toxicity. Thus, for example, the terms "toxic" and "very toxic" were given quantitative definitions based on the $L D_{50} .\left(L D_{50}\right.$ is the median dose lethal to $50 \%$ of a test population.) As a measure of toxicity, the $L D_{50}$ is, at best, an indication of the ability of a substance to cause death following short-term exposure, usually of four days or less and often at unrealistically high concentrations. It tells us nothing about chronic effects or lethality, or about harmful effects of long-term exposure to relatively low concentrations, an important consideration in the human context where exposure may be for a lifetime of up to 100 years or more. Further, it tells us nothing about toxic effects that may be severely disabling, but are not immediately lethal, such as the malformation of limbs in children whose mothers had been prescribed thalidomide during pregnancy. This kind of effect is covered to some extent in labels and safety data sheets by so-called "Risk Phrases," but it must be remembered that absence of a risk phrase does not mean absence of toxicity. It may simply mean absence of relevant data.
In international negotiations for improved chemical safety management and implementation of harmonized laws, linguistic barriers cause problems in mutual comprehension between nations and even between scientific disciplines. Inadequate understanding of exact usage of terms also leads to considerable wastage of time in achieving a common perception of the nature and significance of problems. It is hoped that this book may help to reduce such waste.

Another consequence of misunderstood concepts in toxicology is misclassification of important substances, either prohibiting their use unnecessarily or permitting their use when the risk involved should have been perceived and avoided. A widely misunderstood concept has been "chemical speciation." This concept is tacitly assumed for organic substances, which are, by definition, chemical species of carbon and always subdivided according to structural differences (e.g., as hydrocarbons, aldehydes, ketones, etc.). Carbon compounds have never been given blanket toxicity classification based upon the reasoning that carbon is the main element present and is fundamental to their structure. There is no branch of toxicology called "Carbon Toxicology." On the other hand, there is a branch called "Metal Toxicology," which has tended to classify toxicity of metals by element rather than by species, although different chemical species of metals have vastly different properties, just as do carbon compounds. Thus, there has been blanket elemental classification of metal compounds that have very different chemical and toxicological activities. For example, nickel compounds, as a whole, have been considered to be human carcinogens, largely because of the excess cancers associated with workers in some nickel refineries. In fact, it is likely that only a limited number of nickel compounds are carcinogenic. In the absence of adequate toxicity data relating to most nickel compounds, it has been assumed that nickel cations will be released from all such compounds and that hydrated nickel cations are carcinogenic. So far, the only toxic effect to humans clearly demonstrated for divalent nickel ions in solution is sensitization leading to skin hypersensitivity and associated inflammation.

The action of IUPAC in providing a precise definition of the concept of chemical speciation (Templeton et 
al., 2000) ${ }^{2}$ was the key to opening up a new area of research. This was one of the factors that persuaded the authors of "Concepts in Toxicology" that there might be a need for explanations of other concepts, and that such explanations might provide a stimulus for work at the interface between chemistry and toxicology. Basic concepts in the chemistry and biochemistry of fundamental life processes also require clarification when a precise understanding of them is factored into mechanistic toxicology and risk assessment. Thus, an IUPAC project (2003-001-2-700) was initiated, and the results were published in Pure and Applied Chemistry as an "Explanatory Dictionary of Key Terms in Toxicology (IUPAC Recommendations 2007)."3 "Explanatory Dictionary of Key Terms in Toxicology, Part II," also supported as an IUPAC project (\#2006-020-1-700), will be published in PAC. ${ }^{4}$ Combining the two publications in the form of a book was a logical development that permitted the relationships between the concepts to be further developed and clarified using concept diagrams. This approach is reflected in the structure of the book, which develops concepts starting with fundamental principles of toxicology and risk assessment through the molecular, cellular, and organismal levels to a culmination in ecotoxicology.

References

1. J.H. Duffus, M. Nordberg, D.M. Templeton. Pure Appl. Chem. 79, 1153 (2007).

2. D.M. Templeton, F. Ariese, R. Cornelis, L.-G. Danielsson, H. Muntau, H.P. van Leeuwen, R. Lobinski. Pure Appl. Chem. 72, 1453 (2000).

3. M. Nordberg, J.H. Duffus, D.M. Templeton. Pure Appl. Chem. 79, 1583 (2007).

4. M. Nordberg, J.H. Duffus, D.M. Templeton. Pure Appl. Chem. in press.

\section{Polymer Colloids: From Design to Biomedical and Industrial Applications}

\section{Macromolecular Symposia}

Volume 281, pages 1-212 (July 2009)

Daniel Horák, Jaroslav Kahovec, Jaromir Snupárek (editors)

doi:10.1002/masy.200990017

Every summer, the Institute of Macromolecular Chemistry of the Academy of Sciences of the Czech Republic organizes the Prague Meetings on Macromolecules, which are focused on special topics of polymer chemistry and physics. The papers included in this volume of Macromolecular Symposia were delivered at the 48th Microsymposium "Polymer Colloids: From Design to Biomedical and Industrial Applications," held 20-24 July 2008 in Prague.
The microsymposium, chaired by D. Horák from the Institute and co-chaired by J. Snupárek from the University of Pardubice, focused on new methods in design, development, modeling, characterization, and application of advanced polymer colloids both in industry and life sciences, such as biomedicine and biomedical diagnostics. Biomedical applications and polymers for drug and gene delivery

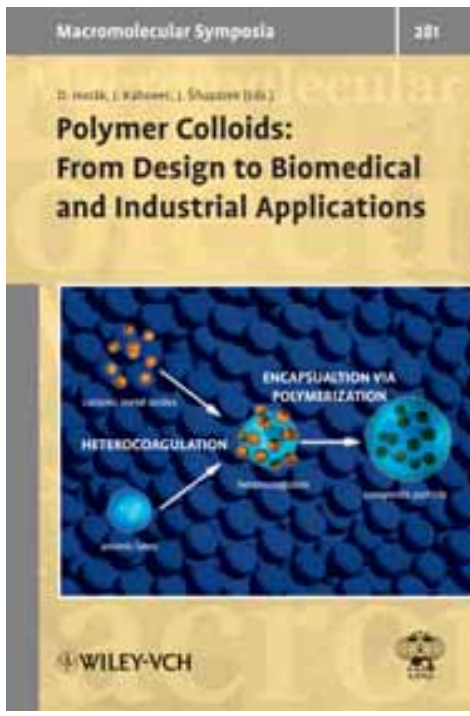
have become an important issue of the host Institute in Prague. The program coverage was granted by a number of top specialists as keynote and main lecturers. Ten keynote lectures and 14 main lectures were presented by invited speakers, while 40 special lectures and 96 poster presentations were provided by other participants. Selected presentations are now reported in an extended form in this volume. 